\title{
Finding Resonance: Adaptive Frequency Oscillators for Dynamic Legged Locomotion
}

\author{
Jonas Buchli*, Fumiya Iida $^{\dagger}$ and Auke Jan Ijspeert* \\ *Biologically Inspired Robotics Group, School of Computer and Communication Sciences \\ Ecole Polytechnique Fédérale de Lausanne (EPFL) \\ INN 241, Station 14, CH-1015 Lausanne, Switzerland \\ Email: jonas@buchli.org, auke.ijspeert@epfl.ch \\ ${ }^{\dagger}$ Artificial Intelligence Laboratory, Department of Informatics \\ University of Zürich \\ Andreasstrasse 15, CH-8050 Zürich, Switzerland \\ Email: iida@ifi.unizh.ch
}

\begin{abstract}
There is much to gain from providing walking machines with passive dynamics, e.g. by including compliant elements in the structure. These elements can offer interesting properties such as self-stabilization, energy efficiency and simplified control. However, there is still no general design strategy for such robots and their controllers. In particular, the calibration of control parameters is often complicated because of the highly nonlinear behavior of the interactions between passive components and the environment.

In this article, we propose an approach in which the calibration of a key parameter of a walking controller, namely its intrinsic frequency, is done automatically. The approach uses adaptive frequency oscillators to automatically tune the intrinsic frequency of the oscillators to the resonant frequency of a compliant quadruped robot. The tuning goes beyond simple synchronization and the learned frequency stays in the controller when the robot is put to halt. The controller is model free, robust and simple. Results are presented illustrating how the controller can robustly tune itself to the robot, as well as readapt when the mass of the robot is changed. We also provide an analysis of the convergence of the frequency adaptation for a linearized plant, and show how that analysis is useful for determining which type of sensory feedback must be used for stable convergence. This approach is expected to explain some aspects of developmental processes in biological and artificial adaptive systems that "develop" through the embodied system-environment interactions.
\end{abstract}

\section{INTRODUCTION}

Although rigid bodies and high-gain motor control for tracking precise trajectories are the basis for the design of traditional robotic systems, there has been an increasing interest of endowing robots with passive dynamics for locomotion behaviors inspired from biological research. In biological locomotion research it has long been realized that the control systems have to work together with the bodies they are controlling. As observed by Marc Raibert, the central nervous system does not control the body, it can only make suggestions [1]. An increasing number of legged robots are, similarly to their biological counterparts [2], [3], endowed with passive dynamics in the form of springs and/or pendulums, and have demonstrated interesting properties such as energy efficiency, self-stabilization, and simple control [4]-[9].

However, an important problem with robots that have passive dynamics is that we do often not know how to properly control them. Because passive dynamics is intrinsically dependent on the physical constraints of the environment, the control architectures have to be highly dynamic and adaptive. For the operation in complex environments, in particular, it is useful for the robot to autonomously find the intrinsic locomotion dynamics. Because the locomotion dynamics is highly dependent on the physical constraints of the body and the environments (e.g. body weight and ground friction), the robot has to constantly and dynamically track and readapt the control parameters to maintain the locomotion dynamics. This is possible since body and sensors yield redundant information and invariants, which a suitable controller can find and exploit. After all, biological systems for locomotion control are extremely adaptive and seem to fulfill these adaptivity requirements.

Therefore, a successful control methodology needs to address the following questions: (1) how can we stabilize the controller-robot systems, (2) control them, and (3) modulate their behavior. These requirements are not different from the ones addressed with traditional linear controller design. For robots with passive dynamics, however additional properties of the controller are needed. Namely, (4) how to find in an autonomous fashion the intrinsic locomotion modalities and, because they can change due to the environment or changes in the body, (5) constantly track and readapt to them.

We propose an approach that addresses the problem of how to automatically and dynamically tune a controller to the, possibly time-varying, properties of a compliant robot body and exploit those properties for locomotion. In particular, we are interested in designing systems made of coupled nonlinear oscillators that are bi-directionally coupled to the robot for the control of locomotion. In this context, it is important for the controllers to be able to track the resonant frequencies of the compliant robot in order to minimize the amount of energy 
that needs to be applied for obtaining locomotion. We do not address the problem of mechanical stability in this work, we are mainly interested in the adaptation problem.

In this article, we implement such an approach by using the adaptive frequency Hopf oscillator [10], [11] for controlling the hopping of a compliant quadruped robot. We designed this oscillator to have several interesting properties such as a structurally stable limit cycle and the ability to tune its intrinsic frequency to the frequency components of arbitrary rhythmic input signals. Particularly interesting are the facts that the system is simple, that it does not require complicated preprocessing and signal analysis techniques, and that it does not require external learning/optimization algorithms (learning is part of the dynamical system).

Oscillators for locomotion control have been presented before (e.g. [6], [12]-[14]), however the automatic, online tuning of their parameters is usually not addressed. In some studies the intrinsic synchronization properties of the oscillators are exploited to modify the gait patterns slightly based on sensory input. These studies use the well-known synchronization properties of nonlinear oscillators, namely that an oscillator receiving a forcing rhythmic input (e.g. from sensors) will oscillate at the frequency of the input as long as the coupling is strong enough and that the intrinsic frequency of the oscillator is not too different from that of the input. This means (1) that synchronization only works when the intrinsic frequency is designed to be close to that of the input, and (2) that frequency of the input is forgotten once the input is removed. In contrast, our approach based on frequency adaptation has three interesting properties: (1) it tunes the intrinsic frequency of the oscillator, not the resulting frequency, (2) the intrinsic frequency converges to the input frequency from any initial condition (ie. even if it is initially very different from it), and (3) after convergence, the intrinsic frequency keeps the value of the input frequency even when the input signal is removed.

In previous work, we have demonstrated in simulation that the adaptive frequency oscillators can be used to adapt to the resonant frequency of the body and initiate locomotion in crawling and hopping robots [10], [15]. In particular, we illustrated how it could adapt to changes in body weight. Here, we extend that work by applying the control architecture to a real robot, the quadruped robot PUPPY II, and by demonstrating that the system can deal with all the noise and time delays of a real system. In addition, we provide a mathematical analysis of the convergence of the frequency adaptation for a linearized plant, and show how that analysis is useful for determining which type of sensory feedback must be used for stable convergence. Interestingly, this analysis explains the experimental facts that the convergence of the adaptation process can be achieved with the sensory information from some sensors but not others (unless the sensor signals are modified). As it becomes clear later in this paper, this finding allows further insights into sensory motor-coupling of adaptive behavior in robots with passive dynamics.

In the next sections, we first describe the adaptive frequency Hopf oscillator and its application to the control of hopping with the quadruped robot (Section II). Experiments show the nice online adaptive property of the controller but also that convergence of the frequency adaptation is obtained with some sensors but not with others (namely with the inertial sensor but not the knee joint angle sensor). We then present a general analysis of a complete system composed of the adaptive oscillator bi-directionally coupled with a linear plant (Section III). We use that analysis to explain the robotic experiments, i.e. how convergence depends on the modality of the sensory information (Section IV). Finally, results are discussed in Section V.

\section{ADAPTIVE FREQUENCY OSCILLATOR ON A REAL ROBOT}

This section explains the control architecture and the initial experimental results with the robotic platform where we use the adaptive frequency Hopf oscillator as a controller. The aim is to motivate the subsequent analysis, more detailed results on the robot experiments will be reported elsewhere. We will see that we need to develop an understanding of adaptive frequency oscillators in feedback loops to explain the experimental results. And hitherto no analysis of adaptive frequency oscillators with feedback loop exists. This article is a first step toward this analysis.

PUPPY II is a small experimental quadruped robot for the investigation of locomotion in robots with passive dynamics. The robot has 4 servo motors in the "hip" joints and passive rotational knee joints with a spring (see Figure 1). It is thus under-actuated and has a very pronounced intrinsic body dynamics in terms of the resonant frequency. The used version of this robot has several sensor capabilites: 3 -axis inertia sensors, touch sensors, knee angle sensors, force sensors under the feet, and an IR distance sensor. From previous research (see [9]) it is known that such a robot has interesting intrinsic locomotion modalities which are closely linked with its passive dynamics. So far however these modes where found by parameter tuning, trial and error, systematic parameter sweeps or other search techniques. Our research aims at finding controllers which are autonomously capable of finding and exploiting such intrinsic locomotion capabilities. We have shown in simulation how very simple dynamical systems can find intrinsic locomotion modalities and elicit them [15]. This robot allows to test the presented approach on a real robot.

The controller consists of an adaptive Frequency Hopf oscillator which has the form [10], [11]:

$$
\begin{aligned}
\dot{r} & =\left(\mu-r^{2}\right) r+\cos \phi y \\
\dot{\phi} & =\omega-\frac{1}{r} \sin \phi y \\
\dot{\omega} & =-\frac{1}{\tau} \sin \phi y
\end{aligned}
$$

where $r, \phi$ and $\omega$ are the state variables for amplitude, phase and frequency respectively. $u=r \cos \phi$ is the oscillatory output signal and $y$ is an input signal that is used to feed sensory input into the oscillator. $u$ (with an appropriate scaling and offset) is used as position setpoint for the servos. If the input $y=0$, this oscillator exhibits a harmonic limit cycle 
a)

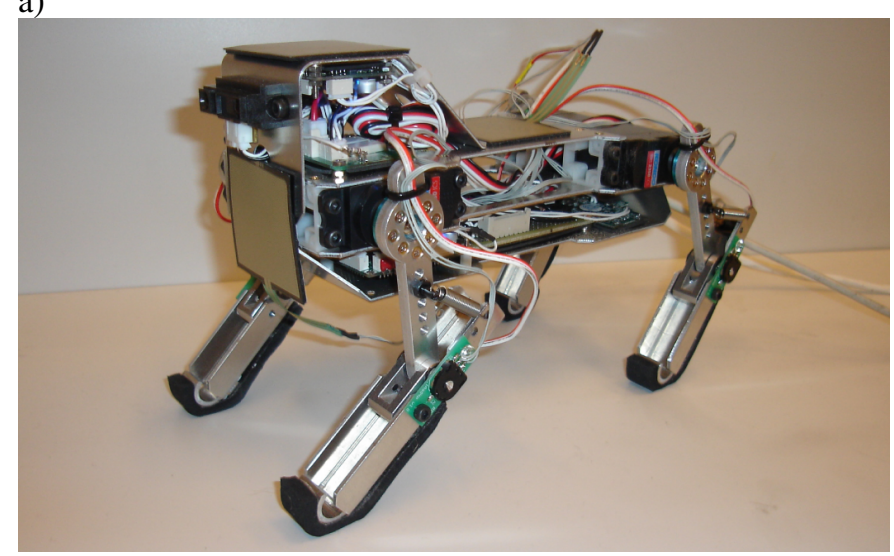

b)

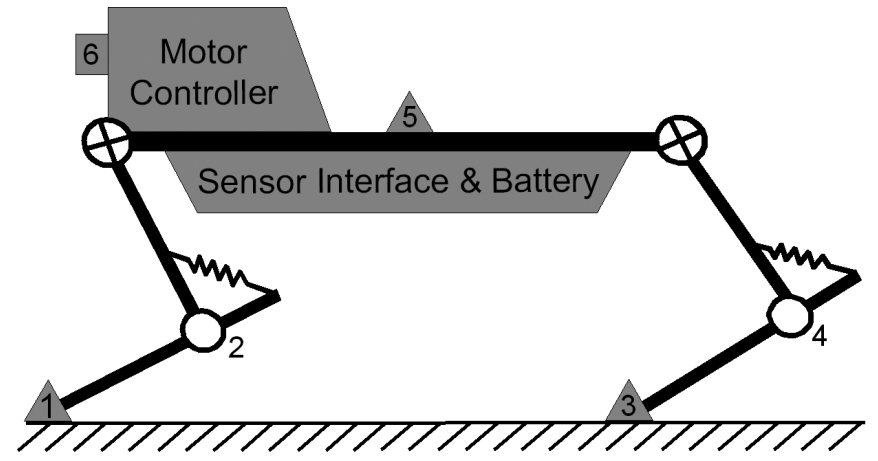

c)

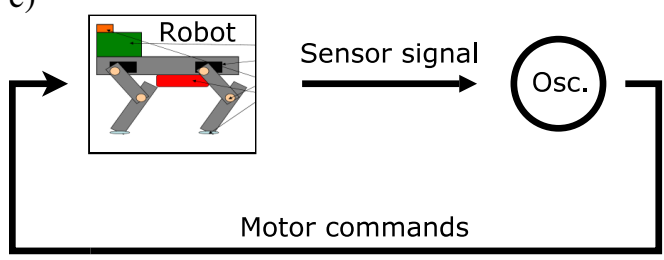

Fig. 1. a) PUPPY II, a robot "dog" with passive dynamics (cf. springs in the knee joints) and several sensor modalities b) Mechanical structure of PUPPY II and sensor placement: 1,3: FSR (Force Sensitive Resistors) 2,4: Potentiometers of the passive joints 5: 3-axis acceleration sensors 6: PSD (Position Sensitive Detector). Circles with a cross denote actuated joints, blank circles denote passive joints. c) Control structure used in the experiments: One of the sensor channels is used to perturb an adaptive frequency Hopf oscillator, the output of the oscillator (a state variable) is used to send motor commands (position control). Thus, this system constitutes a nonlinear feedback loop.

with radius $\sqrt{\mu}$ and frequency $\omega$. If the input is a rhythmic signal the oscillator will tune its frequency to the frequency of the input (see [16] for details). Note that this mechanism is more than mere synchronization, it is real frequency adaptation with a theoretically unlimited basin of attraction. $\tau$ represents the adaptation time constant. Its choice is to a large extent uncritical (here we use $\tau=0.3$ ). The higher this constant the faster the adaptation, but also the larger the fluctuations around the steady state value after convergence. In the presented experiments, one oscillator is used to drive all four legs, i.e. all four legs get the same position set point (i.e. hopping). The aim is to replicate some of the results from [15] on a real robot and then go further and explore more possibilities. As input to the oscillator one of the sensors of the robot is used. The closest match to the setup as in [15] intuitively a)

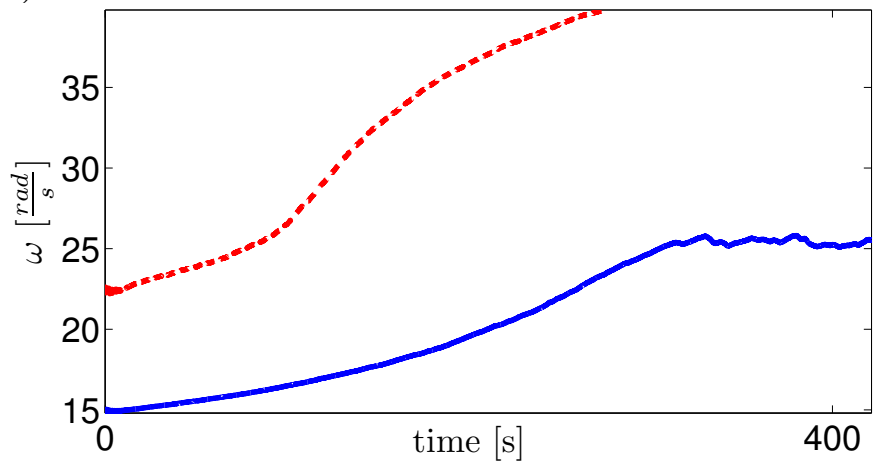

b)

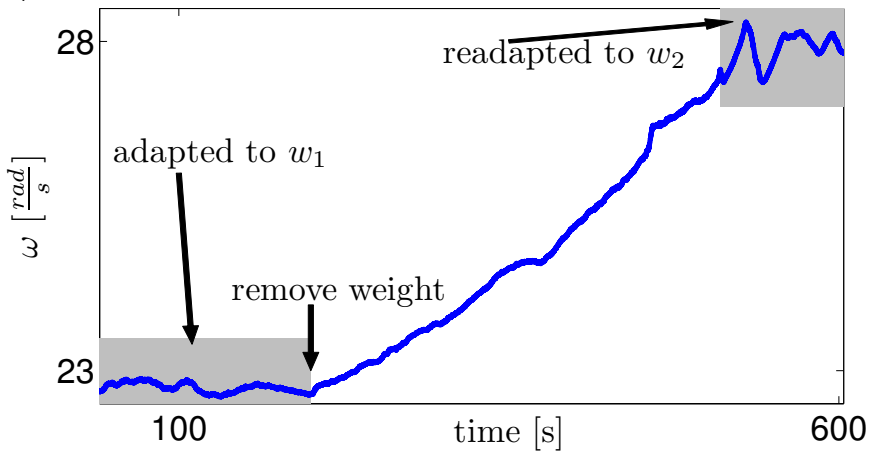

Fig. 2. Results of adaptation experiments with the real-world robot a) The frequency $\omega$ of the oscillator is shown as it changes over time (bold line) adaptation using the z-axis of the inertia sensor: convergence.of the frequency adaptation; (dashed line) adaptation using the knee sensor: divergence of the frequency adaptation with increased rate around the resonant frequency b) An experiment showing the advantage of using online adaptive controllers. The body weight is changed from $w_{1}=0.905 \mathrm{~kg}$ to $w_{2}=0.695 \mathrm{~kg}$. The controller immediately adapts to the changed body property.

is using one of the knee sensors (sensor 2 or 4) as input. In Fig. 2, the result of the experiment with the knee sensor is shown. It is clear that with the knee sensor there is no convergence. This somehow contradicts the intuition from the previous simulation experiments. Thus, a more formal understanding of such a system is needed to understand the convergence properties. Furthermore, if however the inertia sensor (sensor 5, z-axis) is used as an input to the oscillator, a nice convergence is observed, the frequencies to which the oscillator develops indeed correspond to a hopping forward locomotion of the robot (see movies under [17]). Fig. 3 shows an example of the resulting hopping behavior. And, in analogy to the simulation experiments, in Fig. $2 b$ we present the capability of the controller to readapt to change of body properties. This dynamic re-adaptation follows directly from the formulation of the controller as a dynamical system.

The question arises how we can understand the convergence properties and ultimately can we use the insight to improve the convergence properties or design the controller to converge to another body property of our choice.

\section{AdAPtive Hopf OSCILlator With LiNEAR FEEDBACK LOOP}

In order to begin with the analysis of the system, the plant (i.e. the robot) will be modeled by a linear time-invariant 

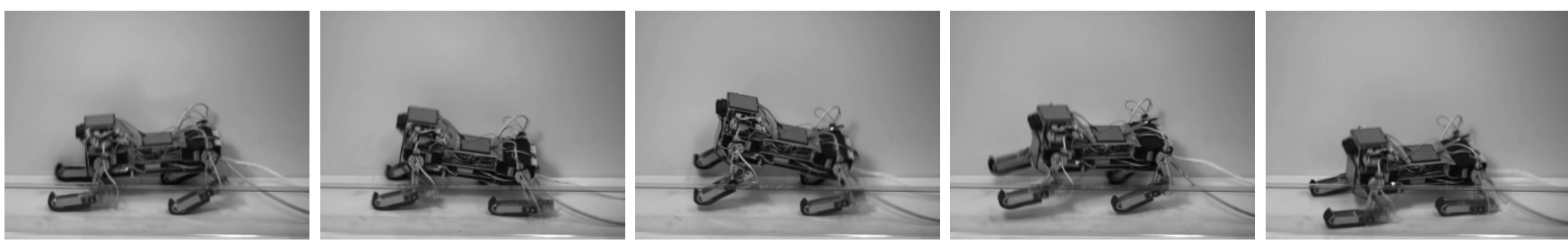

Fig. 3. These snapshots show the hopping movement of the robot after the frequency adaptation has converged. There are moments when the robot has all four legs lifted.

system. This is a simplification but as we will see in the remainder of this article this simplification yields already significant understanding of the mechanism that leads to convergence/divergence.

Thus, in the following we treat the "body" as a linear system. Therefore, lets assume the following systems: First, a linear n-th order SISO (the "robot") of the form

$$
\begin{aligned}
\dot{\mathbf{x}} & =\mathbf{A} \mathbf{x}+\mathbf{B} u \\
y & =\mathbf{C} \mathbf{x}+\mathbf{D} u
\end{aligned}
$$

$\mathbf{A}$ is a $n \times n, \mathbf{B}$ a $n \times 1, \mathbf{C}$ a $1 \times n$ matrix, and $\mathbf{D}$ a scalar. $u$ is the (scalar) input to the linear system and $y$ the (scalar) output.

Second, the adaptive frequency Hopf oscillator as described by Eqs. $1-3$. We set $u=r \cos \phi$. Hence, the Hopf oscillator and the linear systems are connected in a feedback structure through their inputs $y$ (from the linear system to the Hopf Oscillator) and $u$ (from Hopf to the linear system).

As can be shown by linear systems theory, the linear system can not generate other frequencies than already present in the input $u$, it can however modify phase and amplitude of the signal. Therefore, we can write:

$$
y=\operatorname{Ar} \cos (\phi+\alpha)
$$

where $A=|H(s)|$ and $\alpha=\arg (H(s))$. (H(s) is the transfer function of the linear system).

We begin the analysis of the oscillator by writing the phase of the Hopf oscillator perturbed by an input $y$

$$
\dot{\phi}=\omega-\frac{1}{r} \sin \phi y
$$

As outlined above we write $y(t)$ to be the Hopf state amplified by $A$ and rotated by $\alpha$

$$
y(t)=\operatorname{Arcos}(\phi+\alpha)
$$

thus

$$
\dot{\phi}=\omega-\sin \phi A \cos (\phi+\alpha)
$$

Using trigonometric transformations we can write this expression as

$$
\Rightarrow \dot{\phi}=\omega+\frac{1}{2} A[\sin \alpha-\sin (2 \phi+\alpha)]
$$

The results in [11] show that we have a separation of timescale, i.e. the frequency adaptation process works on a timescale much slower then the convergence to the limit cycle with given frequency and radius. Thus, we assume $\omega=$ const and investigate what the observed frequency will be. If this frequency is different then the intrinsic frequency, it should drive the slower adaptation process. $\omega=$ const also implies that $A$ and $\alpha$ are constants $(s=j \omega)$. We evaluate the average effective frequency with the given assumptions:

$$
\bar{\omega}=\frac{1}{2 \pi} \int_{0}^{2 \pi} \omega+\frac{1}{2}[A \sin \alpha-\sin (2 \phi+\alpha)] d \phi
$$

Since by assumption $\omega$ is a constant and therefore $\alpha, A$ are constant, it follows that the first two summands are constants. The last summand is a $2 \pi$-periodic mean-free function, thus we get

$$
\bar{\omega}=\omega+\frac{1}{2} A r \sin \alpha
$$

Let us define $\Delta \omega$ as $\Delta \omega=\bar{\omega}-\omega$, which is a useful notation to discuss convergence:

$$
\Delta \omega=\bar{\omega}-\omega=\frac{1}{2} A r \sin \alpha
$$

From [11]

$$
\Rightarrow \dot{\omega} \approx \frac{1}{2} A^{2} \frac{\omega}{\omega_{F}^{2}-\omega^{2}}
$$

where

$$
\omega_{F}=\omega+\Delta \omega
$$

Assuming $\omega, \omega_{F}>0$ this means if $\Delta \omega>0, \omega$ increases, otherwise it decreases. If $\Delta \omega$ has a zero crossing with a negative slope $\left(\frac{\partial \Delta}{\partial \omega}<0\right)$, there is an attractive region around $\Delta \omega \approx 0$. Therefore, we expect the adaptation of the frequency to have a stable fixed point in this region. Hence, $\Delta \omega$ gives us information on the convergence of the system.

The magnitude of the linear system $A=|H(s)|$ can not be the determinant for convergence since it is positive, thus we have to focus on the phase of the linear system $\alpha=\arg H(s)$. We see that the term $\sin \alpha$ determines the zeros and the sign of $\Delta \omega$, conclusively the phase of the linear system $\alpha=\arg H(s)$ is the determinant for convergence of the adaption process. We will come back to the use of the phase for determining stability in the presentation of the examples. But first we verify numerically the derived approximation.

\section{A. Numerical test of the approximation}

Indeed the result shows that the observed frequency is not the intrinsic frequency. This can be confirmed by computing an FFT on one of the oscillatory state variables $(x, y)$ of the oscillator (data not shown). Here we present another numerical verification of the results.

To test if our assumptions and simplifications are correct we can numerically integrate the differential equation (10) and 


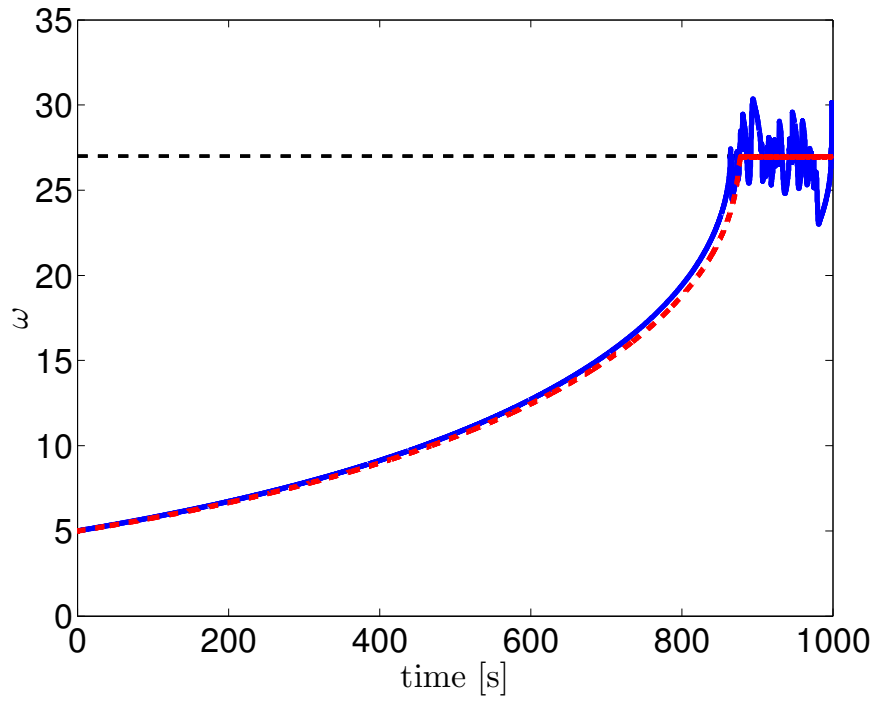

Fig. 4. In this figure we compare the results of the integration of the full system (Eqs.1-5) with the results of the approximation (Eq. 10): (blue) $\omega$ from the integration of the full system, (red dashed) $\omega$ predicted by the approximation. The dashed horizontal line marks the resonant frequency of the linear system.

compare the results with the integration of the full system (Eqs. 1-5). As can be seen in Fig. 4, the approximation yields very close results to the full system for $\omega \neq \omega_{F}$ (using parameters from example A below). Looking at the reasoning in [11] it is clear that the approximation is only correct if $\omega \neq \omega_{F}$. Thus the observed erratic behavior after convergence at $t>860 \mathrm{~s}$ is clearly not a surprise and does not invalidate above results. Furthermore, this erratic behavior is partially due to numeric artifacts by integrating the equation in a straight forward manner with a Runge-Kutta solver.

\section{EXAMPLES OF LINEAR FEEDBACK LOOPS}

Now that we are confident that our reasoning is correct, let us present two examples for a feedback loop with a linear system. The linear system is inspired by a spring mass system. It does however not represent a detailed modeling of the robot.

The two examples will replicate the basic observations in the two presented experiments in Fig. 2. Thus, the goal of these two examples is to show how generic linear systems model for the plant helps to explain the convergence properties of the complete feedback system. We also show that it is very easy to reason for stability once the Bode plots of the linear system are known.

The phase of a linear system is commonly drawn as part of the Bode plot. Bode plots are a very fundamental tool in control engineering for determining stability of feedback loop systems, robust control performance, etc. We can thus exploit all the knowledge experience and tools of working with Bode plots for the analysis of our problem. This means we have a very well developed, rather simple but still very powerful tool to analyze the convergence property of adaptive frequency Hopf oscillators in feedback loops. Furthermore, it can ultimately allow us to design for certain convergence properties, by engineering the phase of the system. This is commonplace in conventional control engineering, thus very well developed techniques exist.

\section{A. Stable at resonance}

Lets assume the following linear system:

$$
\begin{gathered}
\mathbf{A}=\left[\begin{array}{cc}
0 & 1 \\
-\frac{k}{m} & -d
\end{array}\right] \\
\mathbf{B}=\left[\begin{array}{l}
2 \\
0
\end{array}\right] \\
\mathbf{C}=[1,0] \\
\mathbf{D}=0
\end{gathered}
$$

This is 2nd order (e.g. spring mass) system, which possesses a clear resonant frequency at $\omega_{r}=\sqrt{\frac{k}{m}}$. The values of the constants are largely irrelevant for the general result. For the presented data we have chosen $k=27^{2}, m=1$ and $d=$ 0.1 . (Note that we use unit-less constants as their physical interpretation can vary).

In Figure 5, we present the Bode diagrams for the linear systems, and the result of the adaptation of oscillator. As discussed above, the bode plot already gives us a hint for stability of the adaptation process. More precisely we need to look at function $\Delta \omega$, but since this is a second order system, we know that the phase shift is maximum $2 \pi$, thus the phase can only have a single zero crossing. Since sin is a odd function we also know that the sign does not change, thus we can read the stability of the adaptation directly out of the bode plot. In this example we see a negative zero crossing at the resonant frequency, thus we expect the adaptation process converge to the resonant frequency.

In cases where we have a higher order linear system, and also for getting an idea about the quantitative behavior of the convergence, i.e. convergence rates, it is indeed helpful to look at the function $\Delta \omega$ which we obtain in a straight forward manner from the data in the bode plot and inserting into Equation 8.

Thus, in this example we see that the convergence rate should increase and come to a sudden stop. This is indeed the case as can be seen in Fig. 5d, where we show the integration of the full system (Eqs. 1-5).

\section{B. Unstable at resonance}

Now, lets assume the same system as above but we change the coupling from the linear system to the Hopf oscillator (i.e. "change the sensor modality"), by setting

$$
\mathbf{C}=[0,0.1]
$$

i.e. the second state variable of the linear system is now used as input to the Hopf oscillator. Changing only the coupling means, the system has the same resonant frequency as before. Nevertheless, as we will see the adaptation does not converge to this frequency. Again, in Figure 6, we present the Bode diagram. As before by looking at the phase of the linear 

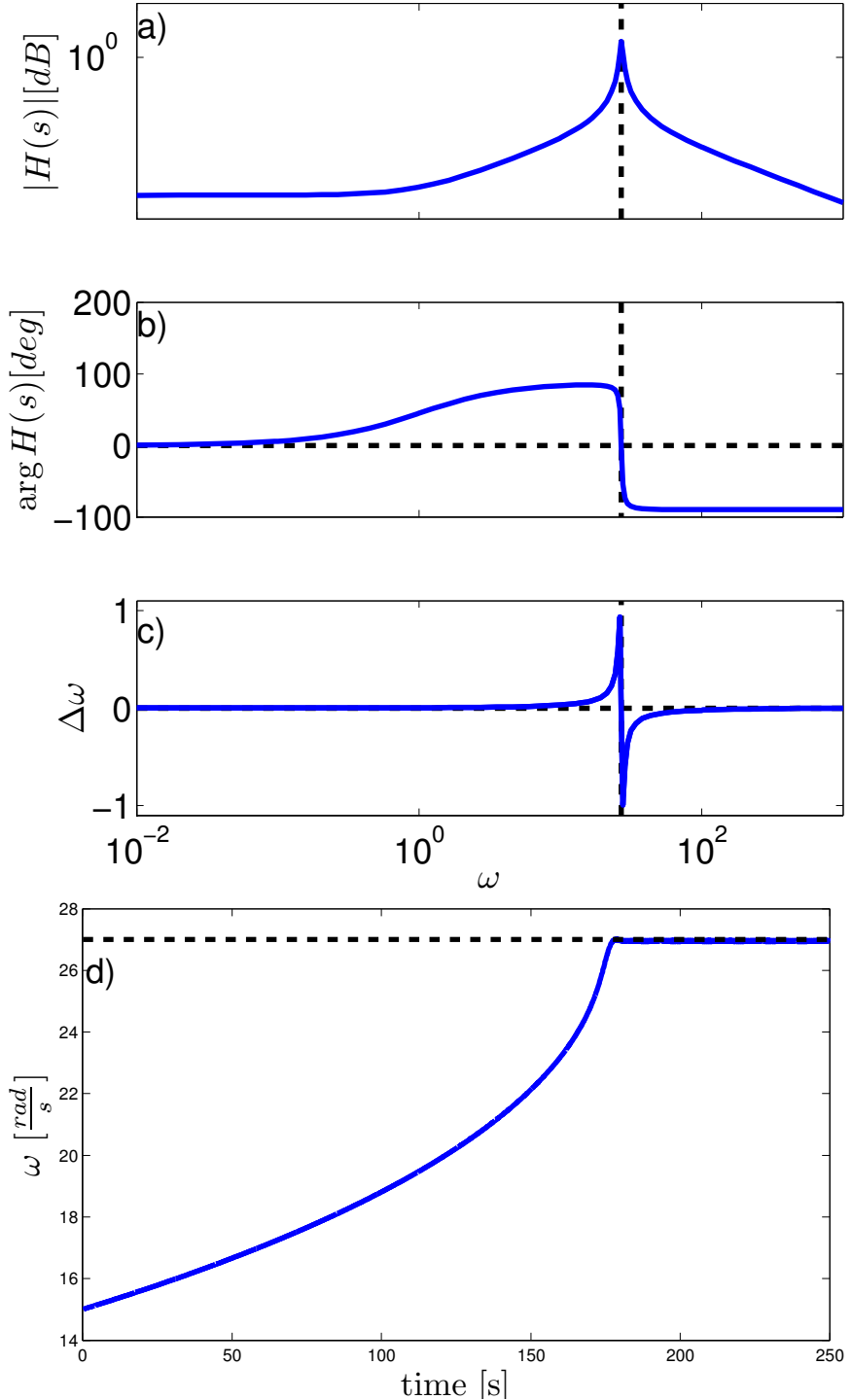

Fig. 5. A linear system for which the convergence is stable at resonance. a,b) Bode plot for $\mathrm{H}(\mathrm{s})$, the dashed line indicates the resonant frequency. Note that the phase has a negative 0 crossing at the resonant frequency, this means the adaptation process has a attractor at the resonant frequency. c) The function $\Delta \omega=\sin (H(s))$. Strictly speaking we need to look at this function to determine stability. d) Time series of the integration of the full system showing the adaptation of the oscillator frequency $\omega$. It is clearly visible how the oscillator frequency adapts to the resonant frequency of the linear system (dashed line), what corresponds to the prediction from the Bode plot and $\Delta \omega$.

system we already gain insight into the expected convergence properties of the frequency adaptation. This time there is no zero crossing, and the rate is positive. Thus, we expect the adaptation process to diverge. Looking at the function $\Delta \omega$, we can see that the convergence rate should be very low, but increases to a peak around the resonant frequency. And indeed, looking at Fig. $6 \mathrm{c}$ this prediction is confirmed. Thus, with the two presented examples we reproduce the observations in the experiments on the real robot that we have convergence with some sensors but not with others (compare Fig. 2 with Figs. $5 / 6 \mathrm{~d})$.
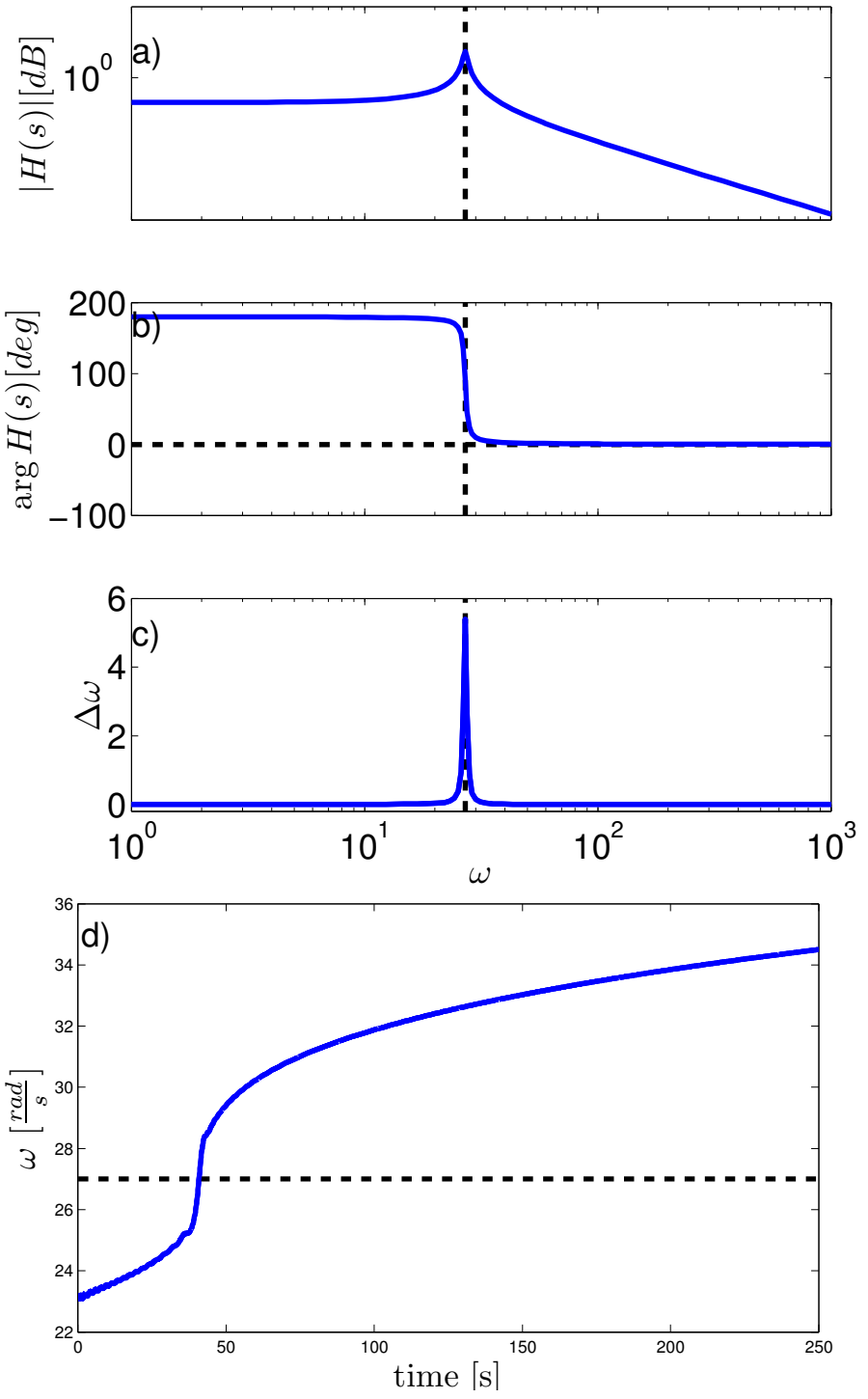

Fig. 6. A linear system for which there is no convergence at resonance. a,b) Bode plot for $\mathrm{H}(\mathrm{s})$, note that the phase has no negative 0 crossing at the resonant frequency, this means the adaptation process has no attractor at the resonant frequency, and especially $\Delta \omega$ (c), is always positive with a distinct peak at the resonant frequency. This means divergence of the oscillator frequency $\omega$ with an increased rate to be expected around the resonant frequency. d) Time series of $\omega$. The prediction is confirmed by the data obtained by the integration of the full system.

\section{Conclusion \& Discussion}

We have presented results on an adaptive controller adapting and exploiting passive dynamics in a robot. This paper showed that the proposed control architecture is able to find the resonance frequency given by the passive dynamics of the realworld robot. The convergence properties are further analyzed by using linear plant models.

The experimental results demonstrated a number of potential advantages of the proposed adaptive frequency oscillator in real-world autonomous adaptive robots. In particular, it is important to note that this architecture requires no preprogrammed models of body dynamics, but it autonomously finds 
adequate control parameters. The experimental results, however, need to be discussed further toward our comprehensive understanding of adaptive control architectures.

The presented analysis has brought us a big step forward in understanding adaptive frequency oscillators in feedback loops. If we compare the convergence behavior of the system with the linear feedback loops (Figs. 5/6d) with the results from the robot experiment (Fig. 2) we see that the linear systems already reproduce the basic features observed in the experiment. We conclude that the linear systems analysis is sufficient to understand basic convergence properties of adaptive frequency Hopf oscillators with feedback loops. We have shown that the phase shift of the linear system is the main determinant of the stability of the adaptation process. Treating the body as linear system presents the simplest case for analysis but yields very important results. The presented work is an important step towards the understanding and designing adaptive controllers for robots with passive dynamics.

Furthermore, this paper also shows how with a description of our adaptive systems in the language of dynamical systems, we can readily explore it with powerful mathematical tools. We have seen that Bode plots are a helpful tool for the analysis but they can also help in the design of the controllers.

Such an adaptive controller is able to autonomously find invariants of the sensor-motor system. The state of the controller reflects these invariants (here the resonant frequency). This states can serve as segmented behavior patterns which can serve as a fundament on which more comprehensive and sophisticated sensory-motor control can build on. Those behavior patterns need to be stable over a certain time otherwise they can not be exploited. In a certain sense such an adaptive controller extracts the slowly varying properties out of the sensor-motor dynamics, and thus represents such meta-stable properties. We can also look at the oscillator as a (very rough) model of the robot of which the parameters are tuned to match the real robot. Loosely speaking finding the correct frequency for the oscillator corresponds to adapting the body schema (the oscillator) to the real body. It is likely that the understanding of such adaptive systems leads to an understanding of the development of cognitive capabilities.

Future work: We have employed linear models for the plant, but the situation on the real robot is a bit more complicated, it needs to be seen to what extent linear models are valid. In order to arrive there, linear models of the sensor-motor modalities of the robot need to be derived. This can be done either theoretically, model-based or by systems identification methods. The prediction of the linear model need then to be checked against the results obtained from the robot.

Furthermore, in future we can explore the design of convergence properties by designing $H(s)$ or adding additional systems in the feedback loop. As an example, phase shift elements (as used to ensure stability of linear feedback loops) could be used to stabilize/destabilize the adaptation process according to some needs. Design techniques for such linear systems are well developed in control theory.

Furthermore, the implications and potential of feedback loops of adaptive frequency oscillators with linear systems is not fully explored and exploited. It is well possible that we can obtain further results from the analysis of this system. A further development is to generalize above system by using MIMO systems in the feedback loop. MIMO systems would correspond to the case where several sensors channels and several actuators are used at the same time.

\section{ACKNOWLEDGMENTS}

The authors would like to thank Ludovic Righetti for helpful discussions.

This research is supported by the Swiss National Science Foundation through a Young Professorship Award to Auke Ijspeert (J.B. \& A.I.) and grant 200021-109210/1 (F.I.).

\section{REFERENCES}

[1] M. Raibert and J. Hodgins, Biologcial Neural Networks in Invertebrate Neuroethology and Robotics. Academic Press, 1993, ch. Legged Robots, pp. 319-354.

[2] R. Alexander, "Elastic energy stores in running vertebrates," Am. Zool., vol. 24, pp. 85-94, 1984.

[3] R. Full and D. Koditscheck, "Templates and anchors: neuromechanical hypotheses of legged locomotion on land." Journal of Experimental Biology, vol. 202, pp. 3325-3332, 1999.

[4] T. McGeer, "Passive dynamic walking," International Journal of Robotics Research, vol. 9, pp. 62-82, 1990.

[5] S. Collins, A. Ruina, R. Tedrake, and M. Wisse, "Efficient Bipedal Robots Based on Passive-Dynamic Walkers," Science, vol. 307, no. 5712, pp. 1082-1085, 2005.

[6] H. Kimura and Y. Fukuoka, "Biologically inspired adaptive dynamic walking in outdoor enviornment using a self-contained quadruped robot: Tekken2," in Proceedings IROS 2004, 2004.

[7] J. Cham, S. Bailer, J. Clark, R. Full, and M. Cutkosky, "Fast and robust: hexapedal robots via shape deposition manufacturing," The International Journal of Robotics Research, vol. 21, no. 10, pp. 869-882, 2002.

[8] M. Raibert, "Trotting, pacing and bounding by a quadruped robot," Journal of Biomechanics, vol. 23, pp. 79-98, 1990.

[9] F. Iida, G. Gomez, and R. Pfeifer, "Exploiting body dynamics for controlling a running quadruped robot." in Proceedings of the 12th Int. Conf. on Advanced Robotics (ICAR05), 2005, pp. 229-235.

[10] J. Buchli and A. Ijspeert, "A simple, adaptive locomotion toy-system," in From Animals to Animats 8. Proceedings of the Eighth International Conference on the Simulation of Adaptive Behavior (SAB'04), S. Schaal, A. Ijspeert, A. Billard, S. Vijayakumar, J. Hallam, and J. Meyer, Eds. MIT Press, 2004, pp. 153-162.

[11] L. Righetti, J. Buchli, and A. Ijspeert, "Dynamic hebbian learning in adaptive frequency oscillators," Physica D, vol. 216, no. 2, pp. 269281, 2006.

[12] J. Collins and S. Richmond, "Hard-wired central pattern generators for quadrupedal locomotion," Biological Cybernetics, vol. 71, no. 5, pp 375-385, 1994

[13] G. Taga, "Emergence of bipedal locomotion through entrainment among the neuro-musculo-skeletal system and the environment," Physica D: Nonlinear Phenomena, vol. 75, no. 1-3, pp. 190-208, 1994.

[14] A. Ijspeert, "A connectionist central pattern generator for the aquatic and terrestrial gaits of a simulated salamander," Biological Cybernetics, vol. 84 , no. 5, pp. 331-348, 2001.

[15] J. Buchli, L. Righetti, and A. Ijspeert, "A dynamical systems approach to learning: a frequency-adaptive hopper robot," in Proceedings of the VIIIth European Conference on Artificial Life ECAL 2005, ser. Lecture Notes in Artificial Intelligence. Springer Verlag, 2005, conference, pp. 210-220.

[16] L. Righetti, J. Buchli, and A. Ijspeert, "From dynamic hebbian learning for oscillators to adaptive central pattern generators," in Proceedings of 3rd International Symposium on Adaptive Motion in Animals and Machines - AMAM 2005. Verlag ISLE, Ilmenau, 2005, p. 45.

[17] A movie of the robot experiment is available at http://birg.epfl.ch. 